\title{
Computer sound card assisted measurements of the acoustic Doppler effect for accelerated and unaccelerated sound sources
}

\author{
T. J. Bensky and S. E. Frey \\ Physics Department, California State University Hayward, Hayward, California 94542
}

\begin{abstract}
An approach to experimentally measuring the speed of a moving object by direct application of the Doppler effect for sound is discussed. The method presented here uses a Windows computer and sound card to record Doppler shifted sound from a moving source. This sound card approach allows for direct acquisition of Doppler shifted sound intensity as a function of time, affording much analytical and pedagogical freedom in undergraduate lab instruction. In addition, the acquisition of such data allows for the experimental study of not only constant velocity sound sources, but of accelerated sound sources as well.
\end{abstract}

\section{INTRODUCTION}

The acoustic Doppler shift is a shifting of a sound's frequency because of relative motion between the sound source and the listener, or observer. The equation for the shift is

$$
f^{\prime}=f \frac{v \pm v_{o}}{v \mp v_{s}},
$$

where $f^{\prime}$ is the Doppler shifted frequency, $f$ is the rest frequency of the sound, $v$ is the speed of sound (in air, 343 $\mathrm{m} / \mathrm{s}), v_{o}$ is the speed of the observer, and $v_{s}$ is the speed of the source. The upper signs are used when the motion of the source is toward the observer, and the lower signs are used when the motion is away.

In the experiment presented here, a $3-\mathrm{W}, 8-\Omega$ speaker is attached to a cart on a (frictionless) air track. A light twin lead wire connects the speaker to a tunable function generator. A microphone is fixed at one end of the air track and is connected to the input of a sound card on a Windows computer. A photogate timer is placed about halfway down the air track. The entire setup is shown in Fig. 1. The sound card is essentially an analog-to-digital converter that records the microphone's response (a voltage) to sound intensity as a function of time. To perform the experiment, the cart is pushed either directly toward or away from the microphone, and the computer is made to record the sound while the cart is moving. The data recorded will capture the speaker's Doppler shifted sound intensity.

The data acquisition step is very straightforward since most Windows computer systems come with a sound card, which we use here as our primary data acquisition instrument. Further, it is not necessary to program custom software to drive and control the sound card, since Windows also comes with a small program called "Sound Recorder" which we have used exclusively here for our data acquisition purposes. This program records voltage input (in this case, from the microphone) into the sound card as a function of time and allows immediate playback or saving to disk. The sample rate of the sound card is software selectable. We chose the "CD-quality" rate of $44100 \mathrm{~Hz}$.

The sound card allows the students to capture sound intensity versus time data. We find this capability to be advantageous over other Doppler measurement methods ${ }^{1-3}$ from an instructional standpoint. It gives the students a direct data set to work with, allowing them to exercise their data analy- sis skills, including scientific use of a computer, data plotting, and graphical interpretation. Additionally, the students can perform their measurements using a variety of audible frequencies (not ultrasonic) and do not have to learn or set up a complicated lab apparatus. The context of the data analysis covers many topics from waves and sound, including frequency, interference, beats, amplitude, and the relationship between the time and frequency domains.

\section{EXPERIMENTAL PROCEDURE}

Using a Pasco PI-9587C function generator connected to the speaker, we select a sound frequency of $1000.0 \mathrm{~Hz}{ }^{4}$ This function generator indicates five significant figures on the selected frequency, and is advertised to be accurate to within $0.01 \%$. To begin the experiment, the cart is placed at rest on the air track near the microphone, and the computer records a few seconds of sound. The data are saved to disk. The cart is then pushed away from the microphone. The computer records the sound while the cart is moving at constant velocity down the air track. Again the data are saved to disk. This is repeated for the cart moving toward the microphone. The time on the photogate is noted for each trial. Three data sets are now available for analysis. The first is sound intensity versus time for the cart at rest. From these data, $f$ can be determined and compared to the frequency set on the function generator. The second and third data sets are sound intensity versus time for the cart moving away from or toward the microphone.

A typical raw sound data recording of a cart pushed away from the microphone is shown in Fig. 2. Region I shows the cart at rest and then being accelerated due to an external push. Region II is the area of interest, and shows the Doppler shifted sound intensity produced while the cart is in motion. The modulations in amplitude seen are beats between the sound coming directly from the speaker and that reflecting off surrounding objects (e.g., the lab bench). Finally, region III shows a large irregular pattern produced when the cart crashes into the end of the track. The region of Doppler shifted data (region II) is extracted from each of the trials for analysis. On the coarse scale of the plot, the $1000.0-\mathrm{Hz}$ frequency oscillations are not visible. The inset shows that the overall data are indeed oscillating. 


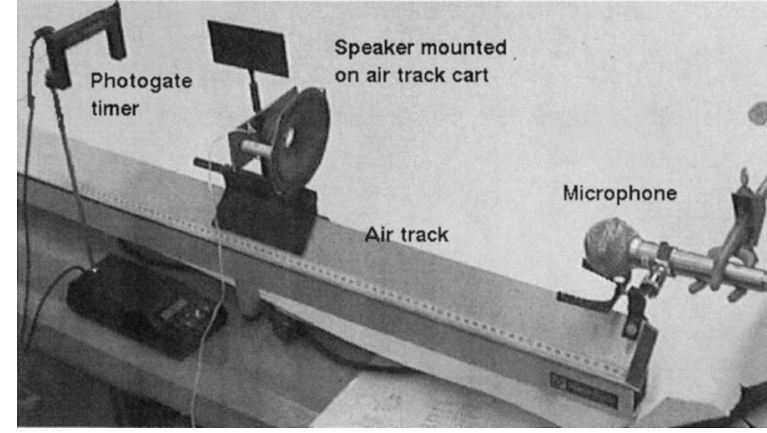

Fig. 1. The experimental apparatus used in this work. Note that the cart can be freely pushed directly toward or away from the microphone. The white wire coming from the speaker is twin-lead, and is connected directly to a function generator.

\section{DATA ANALYSIS}

In this experiment, the observer (microphone) is always at rest, so Eq. (1) will reduce to

$$
f^{\prime}=f \frac{v}{v \mp v_{s}},
$$

when $v_{o}=0$. In the data presented here, the rest frequency on the function generator was set to $1000.0 \mathrm{~Hz}$. This $1000.0-\mathrm{Hz}$ sound is recorded with the sound card, and the frequency is determined by plotting the data using the Microsoft Excel spreadsheet program. We then manually count how many oscillations occur in a given time period. From this we determine an oscillation frequency of $f=1012.9 \mathrm{~Hz}$, which we use in all subsequent calculations, since it reflects the response of our measuring device (the sound card) to sound. This frequency is within $2 \%$ of the setting on the function generator. The velocity of the cart can be found in a variety of ways.

In method one, most suitable for introductory physics laboratories, the Doppler shifted data are plotted. The frequency of these data will be $f^{\prime}$, which is visually determined

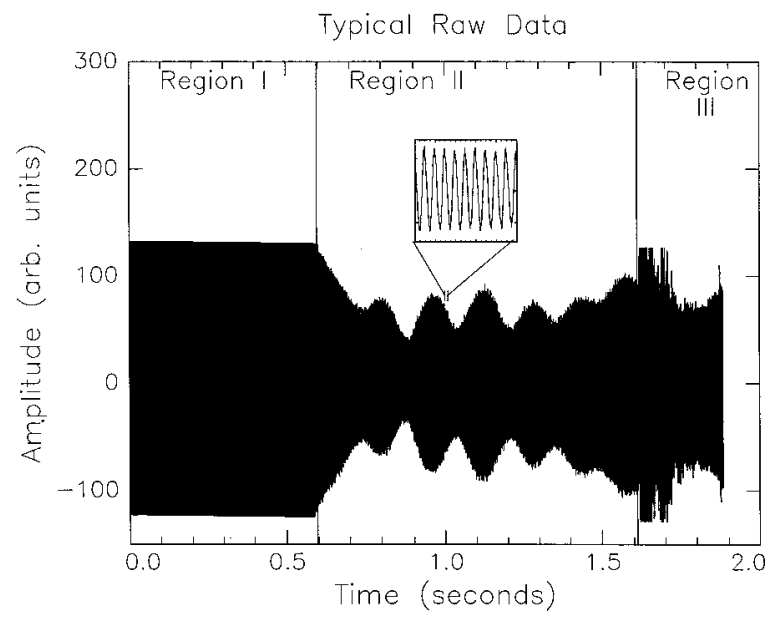

Fig. 2. Typical raw data captured by the sound card for a cart on an air track pushed away from the microphone (observer). Region I contains only the $1000.0-\mathrm{Hz}$ fast oscillation, after the function generator was turned on but before the cart is pushed. The region I/region II boundary is where the cart is pushed. Region II is the sound captured from the speaker mounted on the cart moving at constant velocity. Region III contains the sound of the cart crashing at the other end of the air track.
Table I. Analysis results by visual determination of $f^{\prime} .{ }^{\text {a }}$

\begin{tabular}{ccccc}
\hline \hline Cart direction & $f^{\prime}(\mathrm{Hz})$ & $v_{\text {Doppler }}(\mathrm{m} / \mathrm{s})$ & $v_{\text {photo }}(\mathrm{m} / \mathrm{s})$ & Discr \\
\hline Away & $1009.4 \pm 0.1$ & $1.18 \pm 0.2$ & $1.14 \pm 0.02$ & $3.5 \%$ \\
Toward & $1016.4 \pm 0.1$ & $1.28 \pm 0.2$ & $1.33 \pm 0.02$ & $3.6 \%$ \\
\hline \hline
\end{tabular}

${ }^{a}$ Speed of a moving source found by directly determining $f^{\prime}$ by visual inspection of the Doppler shifted data. Here $v_{\text {Doppler }}$ is the velocity calculated using the Doppler formula and experimental values for $f$ and $f^{\prime} \cdot v_{\text {photo }}$ is the velocity found using a conventional photogate timer.

by graphically determining the time, $\tau_{n}$, between $n$ peaks (or valleys) in the sound data. From this, $f^{\prime}$ can be calculated to be $f^{\prime}=n / \tau_{n}$. Once $f$ and $f^{\prime}$ are known, the velocity of the cart can be calculated by solving Eq. (2) for $v_{s}$ to get

$$
\pm v_{s}=v\left(1-\frac{f}{f^{\prime}}\right) \text {. }
$$

In practice, we also set up a conventional photogate timer that the cart will pass through during the sound recording. This allows for a more direct speed calculation that can be compared with the Doppler version. Results of data analyzed in this manner are summarized in Table I. Here two analysis results are summarized, one for the cart moving toward the microphone, and the other for the cart moving away from the microphone.

Method two uses a slightly more advanced analysis. Here the rest data and Doppler shifted data are "beat" against one another by subtracting one data set from the other. Subtracting two data sets is readily done using Microsoft Excel. Pushing the cart by hand gives a very small Doppler shift; hence $f^{\prime}$ and $f$ are nearly equal, making the data sets ideal candidates for beat analysis. By plotting the difference, the beat frequency, or intensity modulation, can be determined visually. The beat frequency is $f_{b}=f-f^{\prime}$. Since $f_{b}$ is now known experimentally, $f^{\prime}$ can be eliminated from Eq. (3) to find the speed of the cart:

$$
v_{s}=v\left(1-\frac{f}{f-f_{b}}\right) \text {. }
$$

Note that the beat frequency is independent of the phase between the two original signals, so it is not necessary to somehow phase lock the acquisition of the $f$ and $f^{\prime}$ data. A typical beat pattern obtained using this method is shown in Fig. 3. Once such a plot is obtained, the beat frequency can be determined visually. Results of data analyzed in this manner are summarized in Table II, for cart pushes both toward and away from the microphone.

The third and final method for determining the cart's velocity uses a still more advanced approach and is suitable for upper division labs. It employs the Fourier transform to determine $f$ and $f^{\prime}$. Here, the microphone data (time domain data) are loaded into data analysis software and a numerical fast Fourier transform (FFT) is performed. The frequency domain power spectrum will show a single peak centered about the oscillation frequency of the time domain data. Hence, $f$ and $f^{\prime}$ can be found from the peak positions in the FFT spectrum. Once $f$ and $f^{\prime}$ are known, Eq. (3) can be used to determine the velocity of the cart. The results of such analysis are shown in Fig. 4.

Figure 4 contains the results of three separate data sets. The central peak is the FFT of the data taken while the cart is 
Beat Pattern between Rest and Toward Dato

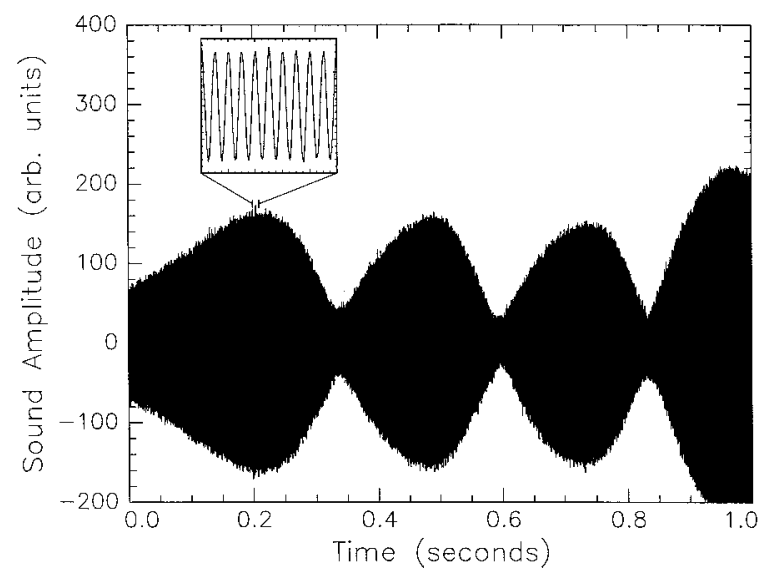

Fig. 3. The beat pattern obtained by subtracting data obtained with the sound source at rest from data obtained with the sound source moving at constant velocity. The fast $\approx 1000-\mathrm{Hz}$ oscillations do not show given the coarse scale of the plot and are shown over a 0.01-s interval by the inset.

at rest and is centered at $\approx 1012 \mathrm{~Hz}$. The peak to the left is obtained for the cart moving away from the microphone, and is centered at $\approx 1015 \mathrm{~Hz}$. The peak to the right is obtained for the cart moving toward the microphone, and is centered at $\approx 1008 \mathrm{~Hz}$. The peak positions are determined visually, and are somewhat subjective, but give results consistent with those reported in Table I.

Precise determination of the peak centers is somewhat subjective. Clearly the peak corresponding to the sound recorded with the cart moving away from the microphone shifts left to a lower frequency. Likewise, the peak corresponding to the cart moving toward the microphone shifts right, to a higher frequency. The peak obtained for the cart at rest does not shift. This is consistent with the principles of the Doppler effect. We find this analysis enlightens advanced students on the correspondence between the time and frequency domains, and how the Fourier transform allows one to go from one domain to the other, all in the context of the readily understandable Doppler effect. Analysis such as this would not be possible without actually being able to acquire and analyze sound intensity versus time data, which is made possible by use of the sound card.

\section{ACCELERATED SOURCES}

A Doppler shift problem from the popular physics text by Serway ${ }^{5}$ involves a tuning fork falling under the constant acceleration due to gravity. Here the tuning fork is an accelerated sound source. The computer and sound card apparatus used here allows for the analysis of such an accelerated source. To study this, the microphone is mounted $\approx 3 \mathrm{~m}$

Table II. Analysis results using the beat frequency. ${ }^{\mathrm{a}}$

\begin{tabular}{ccccr}
\hline \hline Cart direction & $f_{\text {beat }}(\mathrm{Hz})$ & $v_{\text {beat }}(\mathrm{m} / \mathrm{s})$ & $v_{\text {photo }}(\mathrm{m} / \mathrm{s})$ & Discr \\
\hline Away & $3.4 \pm 0.4$ & $1.15 \pm 0.2$ & $1.14 \pm 0.02$ & $<1.5 \%$ \\
Toward & $4.0 \pm 0.4$ & $1.36 \pm 0.3$ & $1.33 \pm 0.02$ & $2.4 \%$ \\
\hline \hline
\end{tabular}

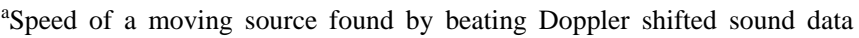
with unshifted data from the source at rest. Here $f_{\text {beat }}$ is the beat frequency determined visually, $v_{\text {beat }}$ is the velocity of the cart determined using the Doppler formula, and $v_{\text {photo }}$ is the velocity found using a conventional photogate timer.

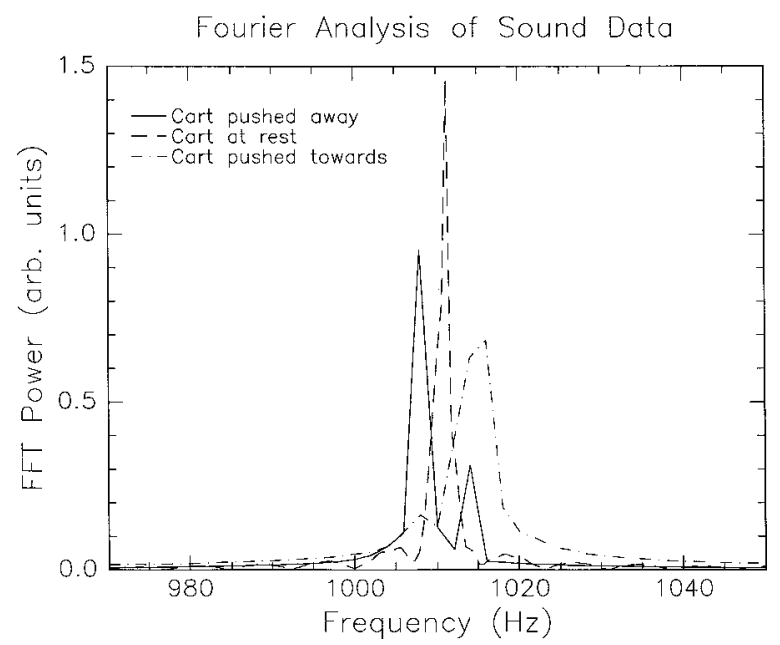

Fig. 4. Fast Fourier transform (FFT) analysis of the time domain sound data for three different data sets. The center peak is obtained by a FFT of sound data recorded while the sound source is at rest. The left peak is obtained when the cart is moving away from the microphone, and the right peak is obtained when the cart is moving toward the microphone.

above the floor. The function generator is again tuned to 1000.0 Hz. The speaker is removed from the air track cart and held just below the microphone; then it is dropped. During the fall, the computer records the sound from the speaker, which is now accelerating away at $9.8 \mathrm{~m} / \mathrm{s}^{2}$. The setup is also reversed, so that the microphone is mounted at floor level and the speaker is dropped toward it from the same height as before. In this manner, data are obtained for a source accelerating both toward and away from the observer.

In this case, the velocity of the source is time dependent, given by $v(t)=g t$, where $g=9.8 \mathrm{~m} / \mathrm{s}^{2}$. This in turn gives rise to a time-dependent, Doppler shifted frequency found by plugging this $v(t)$ into Eq. (2) to get

$$
f^{\prime}=f \frac{v}{v \mp g t}
$$

We find this problem difficult to examine experimentally in the time domain. An observer will hear a time-dependent frequency, but in practice perceiving this is very difficult, if not impossible. We examine the problem instead in the frequency domain, again employing the FFT to make the transformation from time to frequency space.

The results are shown in Fig. 5 for acceleration both toward (middle) and away (bottom) from the observer. For comparison, the frequency spectrum for the source at rest with respect to the observer is shown at the top. Clearly the frequency spectrum in both the toward and away plots have been broadened relative to the spectrum of the source at rest. This spreading is a consequence of the continually shifting frequencies recorded as the speaker accelerates. Note that the toward spectrum shifts and spreads toward higher frequencies, while the away spectrum shifts and spreads toward lower frequencies.

Neglecting air resistance, an object dropped from a height of $\Delta y=3.0 \mathrm{~m}$ will achieve a maximum velocity of $v_{\max }$ $=\sqrt{2 g \Delta y}$, or $v_{\max }=7.6 \mathrm{~m} / \mathrm{s}$. Using $v_{\max }$ and Eq. (2), the maximum observed frequency should be $1034 \mathrm{~Hz}$, when the speaker is accelerating toward the microphone, which is approximately where the power spectrum in the center graph of 


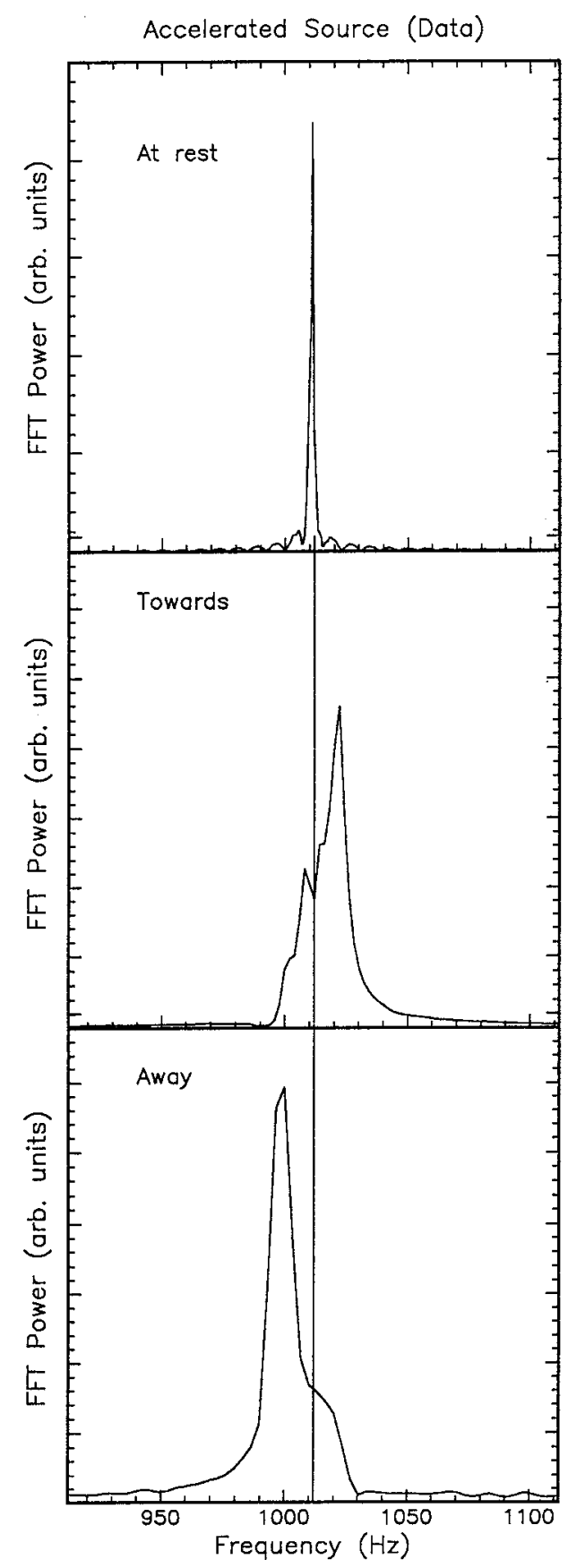

Fig. 5. Fourier spectra for a $1000.0-\mathrm{Hz}$ sound source accelerating at rest (top), accelerating toward the microphone at $9.8 \mathrm{~m} / \mathrm{s}^{2}$ (middle), and accelerating away from the microphone (bottom) at $9.8 \mathrm{~m} / \mathrm{s}^{2}$. The initial source/ microphone separation distance is $3.0 \mathrm{~m}$.

Fig. 5 begins to approach zero. Likewise, the minimum frequency observed when the speaker is accelerating away from the microphone will be $990 \mathrm{~Hz}$, which is approximately where the power spectrum in the bottom graph of Fig. 5 begins to approach zero.

The data obtained can be explained further by modeling the sound detected by the microphone as follows. The sound oscillation is a sine wave with a time-dependent frequency [see Eq. (5)], and an amplitude that varies with the square of the distance from the source, $y=1 / 2 g t^{2}$. If $I_{t}(t)$ is the intensity of sound recorded by the microphone when the source is moving toward the microphone, then we define

$$
I_{t}(t)=\frac{g^{2} t^{4}}{4} \sin \left(\frac{2 \pi f t}{v-g t}\right) .
$$

Likewise, if $I_{a}(t)$ is the intensity of sound recorded by the microphone when the source is moving away from the microphone, then we define

$$
I_{a}(t)=\frac{4}{g^{2} t^{4}} \sin \left(\frac{2 \pi f t}{v+g t}\right)
$$

To model our experiment, data points from both $I_{t}(t)$ and $I_{a}(t)$ as a function of time are generated on a computer from $t=0.05$ to $t=0.78 \mathrm{~s}$. Data are generated over this range to avoid the singularity at zero in Eq. (7), and to stop at the time when an object dropped from $3 \mathrm{~m}$ would hit the ground. Additionally, a pure $1012.0-\mathrm{Hz}$ sine wave is generated for comparison. The FFT of the three generated data sets is computed and shown in Fig. 6. From this simple model, good qualitative agreement is found between the theory and data, assuming an arbitrary overall multiplicative factor in FFT power. The spectrum for the rest data and the theory both result in a narrow peak centered at the rest frequency of the sound. The toward and away spectra both show broadened peaks. The peak centers shift in accordance with the Doppler effect.

The discrepancy in the precise peak positions between theory and data is primarily due to amplitude effects in the time domain data. In our model, we have assumed an inverse-square dependence, but this does not appear to agree entirely with the data in region II, shown in Fig. 2, where we observe very little decline in amplitude as the cart moves away from the microphone.

In the model, the inverse-square dependence causes the oscillations in time to be dominated by large amplitude oscillations near the rest frequency in the away case (at small times, just as the speaker begins to fall). This is responsible for the dominant peak at $\approx 1012 \mathrm{~Hz}$. In the toward case, the time domain model is dominated by large amplitude, higher frequency oscillations, where the accelerated source has its largest velocity at large times, just as the source is about to strike the ground. This skews the frequency spectrum toward the larger frequencies.

\section{CONCLUSIONS}

In summary, we have presented a new, modern, and inexpensive method for performing experiments in the undergraduate lab involving the Doppler shift for sound. The method allows for direct acquisition of sound intensity as a function of time, suitable for further analysis by students. Data analysis can be performed to determine the velocity of a sound source using the Doppler effect at a variety of instructional levels. Additionally, it is possible to experimentally study the Doppler effect for an accelerated source of sound.

The commonly found computer sound card has proven very useful in the scientific measurements presented here. Sound recording software that comes with the Windows operating system ${ }^{6}$ eliminates the need to create or purchase custom data acquisition software. 


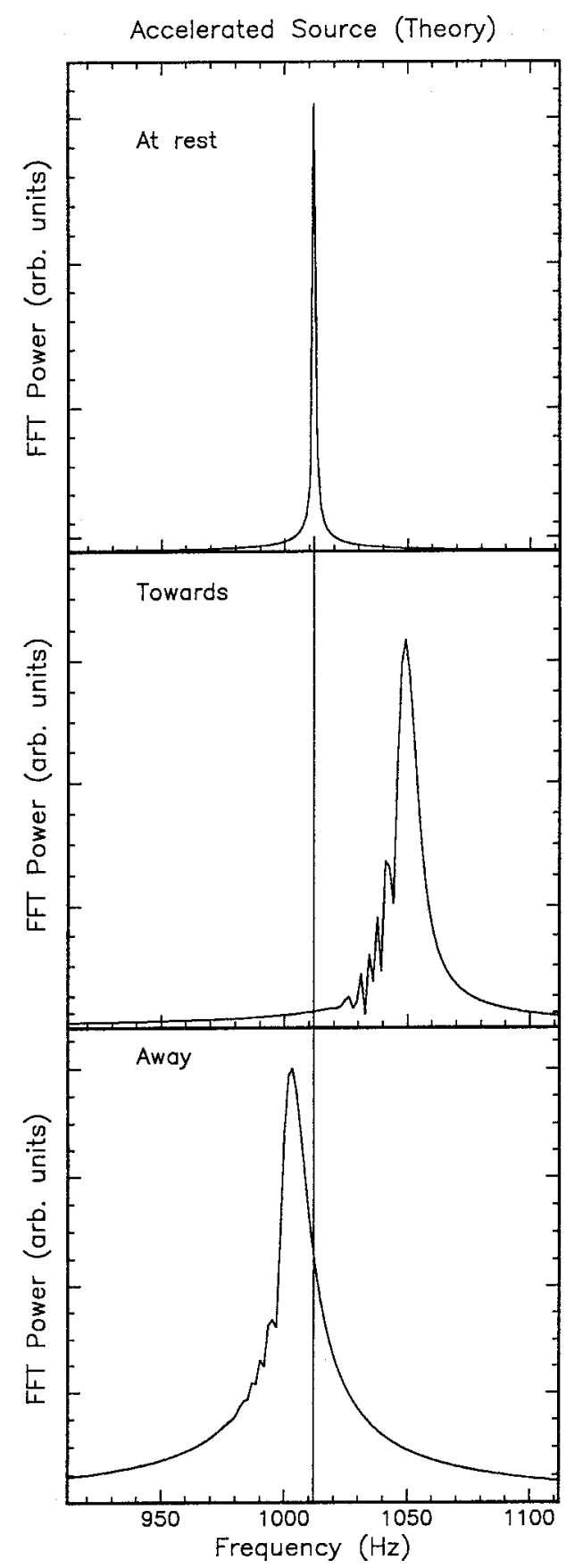

Fig. 6. Calculated Fourier spectra for a $1000.0-\mathrm{Hz}$ sound source accelerating at rest (top), accelerating toward the microphone at $9.8 \mathrm{~m} / \mathrm{s}^{2}$ (middle), and accelerating away from the microphone (bottom) at $9.8 \mathrm{~m} / \mathrm{s}^{2}$.

\section{ACKNOWLEDGMENTS}

We wish to thank the CSUH Office of Research and Sponsored Programs for their support of this project, and M. Ali of the CSUH Physics Department Service Center for help in constructing various parts of this apparatus. T. B. wishes to thank R. Good and P. Carrazana of the CSUH Physics Department for their help in proofreading this paper.

\section{APPENDIX: COMPUTER ISSUES}

The use of a computer sound card has been a great benefit in simplifying the data acquisition in this work. There is one difficulty with this technique, however. Typical recording

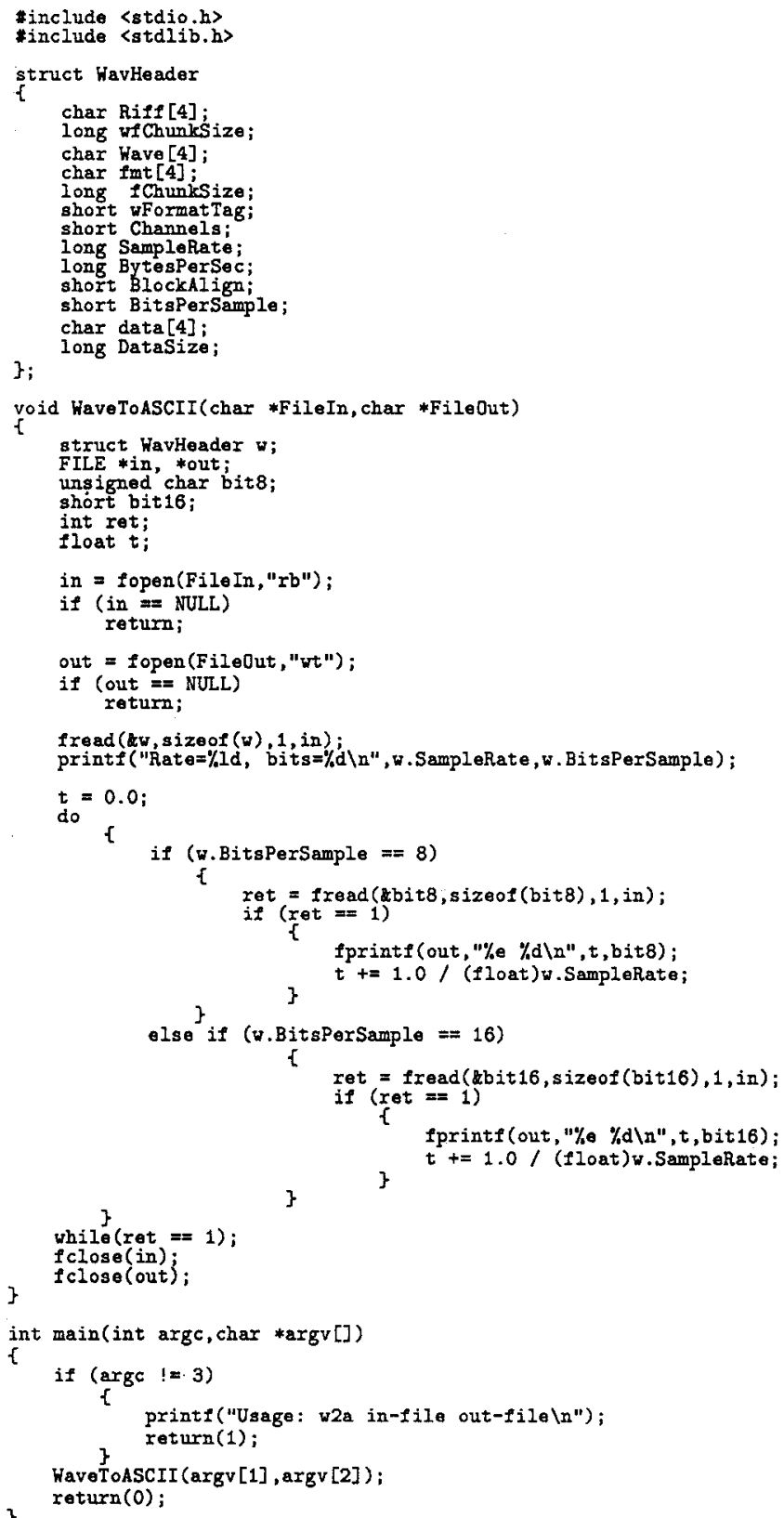

Fig. 7. A C program that converts a WAV sound file into a two-column text file.

software, such as the "Sound Recorder" that comes with Windows, saves sound data in the "WAV" format, a file format for sound in the Windows environment. ${ }^{7}$ Apart from a file header at the beginning, a WAV file is simply a binary file containing the individual data samples read from the sound card as a function of time. For the sake of data analysis later, a WAV file must be converted into a text file, suitable for importing into data analysis software, such as Microsoft Excel. We include a short, 82 line $\mathrm{C}$ program here that will take as input a WAV file, and will output a twocolumn text file. The first column will be time, starting at zero, in increments of the inverse sample rate of the data, as read from the header of the WAV file. The second column will be the value read from the sound card at that particular time. The source code can also be obtained via email by contacting TB. The complete program that performs this WAV file translation is shown in Fig. 7. This program should 
work and compile with any C-compiler (Borland, Microsoft, Gcc, etc.).

${ }^{1}$ A. J. Cox and Joel J. Peavy, "Quantitative measurements of the acoustic Doppler effect using a walking speed source," Am. J. Phys. 66, 11231124 (1998).

${ }^{2}$ R. C. Nerbun, Jr. and R. A. Leskovec, "Quantitative measurement of the Doppler shift at an ultrasonic frequency" Am. J. Phys. 44, 879-881 (1976).

${ }^{3}$ George Barnes, “A Doppler experiment," Am. J. Phys. 42, 905-909 (1974).
${ }^{4}$ We chose $1000 \mathrm{~Hz}$ for a practical reason: It represents about the highest frequency that students found tolerable to listen to while performing this lab. As a referee for this paper pointed out however, the use of higher $(5000-10000 \mathrm{~Hz})$ frequencies could lead to better beat frequency determination and cleaner Fourier spectra.

${ }^{5}$ R. A. Serway, Physics for Scientists and Engineers (Saunders College Publishing, Philadelphia, PA, 1996), 4th ed., p. 495, Problem 40.

${ }^{6} \mathrm{We}$ found several other sound recording packages at the web sites http:// www.download.com and http://www.shareware.com.

${ }^{7}$ C. Petzold, Programming Windows (Microsoft Press, Redmond, WA, 1996), 5th ed., Chap. 22. 